\title{
Conductivity of epitaxial and CVD graphene with correlated line defects
}

\author{
T. M. Radchenko, ${ }^{1}$ A. A. Shylau, ${ }^{2}$ and I. V. Zozoulenko ${ }^{3}$ \\ ${ }^{1}$ Deptartment of Solid State Theory, Institute for Metal Physics of NASU, \\ 36 Acad. Vernadsky Blvd., UA-03680 Kyiv, Ukraine \\ ${ }^{2}$ Center for Nanostructured Graphene (CNG), Department of Micro- and Nanotechnology, \\ DTU Nanotech, Technical University of Denmark, DK-2800 Kongens Lyngby, Denmark and \\ ${ }^{3}$ Laboratory of Organic Electronics, ITN, Linköping University, SE-60174 Norrköping, Sweden
}

(Dated: July 7, 2021)

\begin{abstract}
Transport properties of single-layer graphene with correlated one-dimensional defects are studied using the time-dependent real-space Kubo-Greenwood formalism. Such defects are present in epitaxial graphene, comprising atomic terraces and steps due to the substrate morphology, and in polycrystalline chemically-vapor-deposited (CVD) graphene due to the grain boundaries, composed of a periodic array of dislocations, or quasi-periodic nanoripples originated from the metal substrate. The extended line defects are described by the long-range Lorentzian-type scattering potential. The dc conductivity is calculated numerically for different cases of distribution of line defects. This includes a random (uncorrelated) and a correlated distribution with a prevailing direction in the orientation of lines. The anisotropy of the conductivity along and across the line defects is revealed, which agrees with experimental measurements for epitaxial graphene grown on SiC. We performed a detailed study of the conductivity for different defect correlations, introducing the correlation angle $\alpha_{\max }$ (i.e. the maximum possible angle between any two lines). We find that for a given electron density, the relative enhancement of the conductivity for the case of fully correlated line defects in comparison to the case of uncorrelated ones is larger for a higher defect density. Finally, we study the conductivity of realistic samples where both extended line defects as well as point-like scatterers such as adatoms and charged impurities are presented.
\end{abstract}

PACS numbers: 81.05.ue, 72.80.Vp, 72.10.Fk

\section{INTRODUCTION}

As for all crystalline solids, the presence of a certain amount of disorder in graphene,- the thinnest known material nicknamed as a "miracle material" due to its superior properties, $\frac{1}{-}$ is dictated by the second law of thermodynamics. Defects, playing a role of disorder, are always present in graphene samples due to the imperfection of the fabrication processes, and even can be not always stationary, migrating with a certain mobility gov-

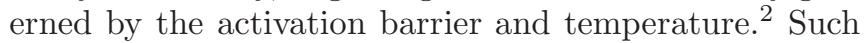
migration and relaxation to the equilibrium state as well as the features of the growth technology can result in a correlation in the configuration of point or/and line defects. The effect of the spatial correlations of point defects on the transport properties of graphene is currently under debate. A recent observation of the temperature enhancement of the conductivity of exfoliated graphene was attributed to the effect of dopant correlations $\frac{3}{2}$ This conclusion was based on the semi-classical predictions relying on the standard Boltzmann approach within the

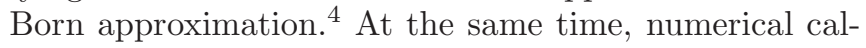
culations within the time-dependent real-space KuboGreenwood formalism showed ${ }^{5}$ that correlation in the spatial distribution of short- and long-ranged point defects do not lead to any enhancement of the conductivity in comparison to the uncorrelated case.

In epitaxial graphene the surface steps caused by substrate morphology are spatially correlated and act as line scatterers for the charge carriers ${ }^{6}$ Epitaxial graphene films grown on $\mathrm{SiC}^{6.7}$ (by $\mathrm{SiC}$ decomposition) or on $\mathrm{Ru}^{8}$ (by CVD method) comprise two distinct self-organized periodic regions of terrace and step, leading to ordered graphene domains $\stackrel{8}{-}$ Step edges, as well as single defects, can be visualized by the Kelvin probe force microscopy $(\mathrm{KPFM}) \stackrel{7,9}{,}$ the scanning tunneling microscopy (STM) or the atomic force microscopy (AFM). For instance, a width of the steps, observed from the AFM images, remained nearly constant, about $10 \mathrm{~nm} ;-6$ while the step heights identified from the KPFM and the STM varied respectively from 0.09 up to $0.75 \mathrm{~nm}^{7}$ and from 0.5 up to $1.5 \mathrm{~nm} \stackrel{10}{=}$ Scanning tunneling potentiometry (STP) measurements of a local electric potential (as current flows through a graphene film) demonstrate 10 that local perturbations caused by the substrate atomic steps are critical to transport in graphene. The STP potential measurements are now possible not only on micro- and macroscopic length scales, 10 but on mesoscopic scale as well,,$\frac{11}{r}$ where the quantum nature of transport manifests itself directly.

Experimental measurements show an increase of the resistance with the step density, 12 the step heights,,$\stackrel{10}{=}$ and the step bunching. 13 Also, an anisotropy of the conductivity in the parallel and perpendicular directions to the steps is revealed, which is due to higher defect abundance in the step regions $\frac{6,14}{}$ Substrate steps alone increase the resistivity in several times relative to a perfect terrace, 10 with the ratio of the estimated electron mobilities in the terrace and step regions being about 10:1 $\underline{6}$ Despite the strong curvature of graphene in the vicinity of steps, a structural deformation contributes only little to electron scattering 15 For the SiC substrate, the 
dominant scattering mechanism is provided by the sharp potential variations in the vicinity of the step due to the electrostatic doping from the substrate strongly coupled with graphene in the step regions $\underline{15}$

The anisotropic charge transport have been also revealed in CVD-grown graphene due to the parallel orientation of the quasi-periodic nanoripples 16 The charged line defects are believed to represent the limiting scattering mechanism of the electronic mobility in CVD graphene $\underline{17-20}$ In this case the line defects correspond to grain boundaries separating grains of different (or the same) crystal orientations 21 or quasi-periodic nanoripples originated from the metal substrate. 16 In contrast to the grain boundaries in the CVD-grown graphene exhibiting mainly (but not always) 16 a random network of lines, the substrate atomic steps acting as the line defects in epitaxial graphene manifest correlation in their orientation and even can be almost parallel to each other as a result of epitaxial growth ${ }^{6-8}$ Taking into account that the charged line defects govern the electrical transport in CVD-grown graphene, the atomic-stepped line defects in epitaxial graphene are also expected to affect strongly its transport properties and even govern them especially if the correlation can be controlled.

The main aim of this paper is therefore to investigate the influence of the orientational correlation of the extended line defects on the conductivity of graphene. We do this numerically, utilizing the quantum mechanical time-dependent real-space Kubo method ${ }^{5,19,22-27}$ allowing us to study graphene sheets approaching the realistic dimensions of millions of atoms.

The paper is organized as follows. In Sec. II, the basic model of the system at hand including a model potential for one-dimensional (1D) defects and the basics of the numerical Kubo method are formulated. Section III presents and discusses the obtained numerical results. Finally, the conclusions of our work are given in Sec. IV.

\section{TIGHT-BINDING MODEL AND KUBO-GREENWOOD FORMALISM}

We model electron dynamics in graphene using a standard $p$-orbital nearest-neighbor tight-binding Hamiltonian defined on a honeycomb lattice,, $28-30$

$$
\hat{H}=-u \sum_{i, i^{\prime}} c_{i}^{\dagger} c_{i^{\prime}}+\sum_{i} V_{i} c_{i}^{\dagger} c_{i}
$$

where $c_{i}^{\dagger}$ and $c_{i}$ are the standard creation and annihilation operators acting on a quasiparticle on the site $i$, the summation is carried out over all nearest-neighbor sites $i$ and $i^{\prime}$ of graphene lattice, $u=2.7 \mathrm{eV}$ is the hopping integral for the neighboring $\mathrm{C}$ atoms $i$ and $i^{\prime}$ with the distance $a=0.142 \mathrm{~nm}$ between them, and $V_{i}$ is the on-site potential describing scattering by defects. For the case of epitaxial graphene on $\mathrm{SiC}(0001)$ the scattering is related to an electrostatic doping at the steps from the interface state on the Si-terminated substrate which has a metallic character with a high density of interface states $, 15,31,32$ Considering this state as a charged line following the step, the effective potential for electron in graphene can be calculated in an analytical form using the Thomas-Fermi (TF) approximation 17.18 The effective TF potential is expressed via the cosine and sine integral functions, and can be well fitted by the Lorentzian-shaped function. 18 If there are $N_{l} 1 \mathrm{D}$ scatterers in graphene, we model them by the effective long-range Lorentzian-type potential,

$$
V_{i}=\sum_{j=1}^{N_{l}} U_{j} \frac{A}{B+x_{i j}^{2}},
$$

where $U_{j}$ is a potential height, $x_{i j}$ is a distance between the site $i$ and the line $j$, and the fitting parameters $A$ and $B$ depend on the considered charge carrier (electron) densities 18 In the present study, we consider two cases, namely, symmetric (attractive and repulsive, $V \gtrless 0$ ), and asymmetric (repulsive for electrons, $V>0$ ) scattering potentials, where $U_{j}$ are chosen randomly in the ranges $[-\Delta, \Delta]$ and $[0, \Delta]$, respectively, with $\Delta$ being the maximum potential height. Figure 1 illustrates the potential shape for both symmetric $(V \gtrless 0)$ and asymmetric $(V>0)$ cases, where, to characterize the relative positions of lines, we introduced correlation angle $\alpha_{\max }-$ the maximum possible angle between any two lines. Note that an electrostatic doping discussed above represents one of possible mechanisms leading to the enhanced scattering at the steps in epitaxial graphene. Other sources of scattering can include, for example, trapped silicon atoms that tend to aggregate at the step edges $\stackrel{14}{=}$ We therefore consider our scattering potential, Eq. (2), as a phenomenological model which with a proper adjustment of the parameters can describe both short- and long-range scattering caused by the presence of $1 \mathrm{D}$ defects.

To calculate numerically the dc conductivity $\sigma$ of epitaxially- or CVD-grown graphene layers with 1D extended defects, the real-space order- $N$ numerical implementation within the Kubo-Greenwood formalism is employed, where $\sigma$ is extracted from the temporal dynamics of a wave packet governed by the time-dependent Schrödinger equation,$\underline{522-27}$ This is a computationally efficient method scaling with the number of atoms in the system $N$, and thus allowing treating very large graphene sheets containing many millions of $\mathrm{C}$ atoms.

A central quantity in the Kubo-Greenwood approach is the mean quadratic spreading of the wave packet along the $x$-direction at the energy $E, \Delta \hat{X}^{2}(E, t)=\langle(\hat{X}(t)-$ $\left.\hat{X}(0))^{2}\right\rangle$, where $\hat{X}(t)=\hat{U}^{\dagger}(t) \hat{X} \hat{U}(t)$ is the position operator in the Heisenberg representation, and $\hat{U}(t)=e^{-i \hat{H} t / \hbar}$ is the time-evolution operator. Starting from the KuboGreenwood formula for the dc conductivity, 33

$$
\sigma_{x x}=\frac{2 \hbar e^{2} \pi}{\Omega} \operatorname{Tr}\left[\hat{v}_{x} \delta(E-\hat{H}) \hat{v}_{x} \delta(E-\hat{H})\right],
$$



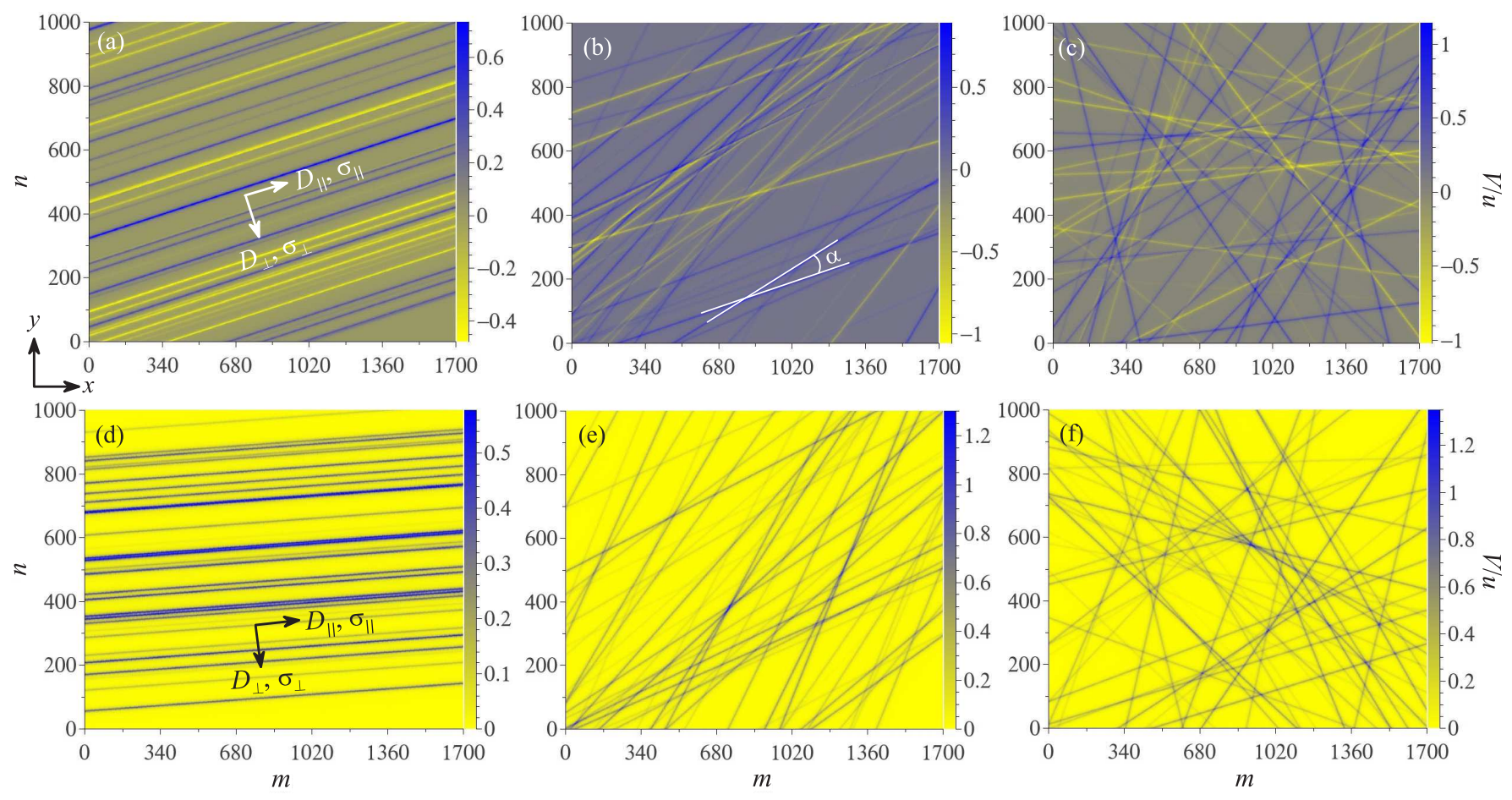

Figure 1: (Color online) Distributions of (a)-(c) symmetric, $V \gtrless 0$, and (d)-(f) asymmetric, $V>0$, scattering potentials, Eq. (2), for a representative configuration of 50 orientationally correlated line defects with different correlation angles $\alpha_{\text {max }}$ (the maximal possible angle between any two lines): $0^{\circ}$ (a), (d); $45^{\circ}$ (b), (e); $90^{\circ}$ (c), (f). Note, that $\alpha_{\max }=0^{\circ}$ and $\alpha_{\max }=90^{\circ}$ correspond to the cases of parallel and random (totally uncorrelated) lines, respectively. The graphene lattice size is $m \times n=1700 \times 1000$ sites corresponding to $210 \times 210 \mathrm{~nm}$. The maximum potential height $\triangle=0.25 u$.

where $\hat{v}_{x}$ is the $x$-component of the velocity operator, $E$ is the Fermi energy, $\Omega$ is the area of the graphene sheet, and factor 2 accounts for the spin degeneracy, the conductivity can then be expressed as the Einstein relation,

$$
\sigma \equiv \sigma_{x x}=e^{2} \tilde{\rho}(E) \lim _{t \rightarrow \infty} D(E, t),
$$

where $\tilde{\rho}(E)=\rho / \Omega=\operatorname{Tr}[\delta(E-\hat{H})] / \Omega$ is the density of sates (DOS) per unit area (per spin), and the time-dependent diffusion coefficient $D(E, t)$ is related to $\Delta \hat{X}^{2}(E, t)$,

$$
\begin{aligned}
D(E, t) & =\frac{\left\langle\Delta \hat{X}^{2}(E, t)\right\rangle}{t} \\
& =\frac{1}{t} \frac{\operatorname{Tr}\left[\left(\hat{X}_{H}(t)-\hat{X}(0)\right)^{2} \delta(E-\hat{H})\right]}{\operatorname{Tr}[\delta(E-\hat{H})]} .
\end{aligned}
$$

It should be noted that in the present study we are interested in the diffusive transport regime, when the diffusion coefficient reaches its maximum. Therefore, following Refs. 25, 26, we replace in Eq. (4) $\lim _{t \rightarrow \infty} D(E, t) \rightarrow$ $D_{\max }(E)$, such that the dc conductivity is defined as

$$
\sigma=e^{2} \tilde{\rho}(E) D_{\max }(E) .
$$

The DOS is also used to calculate the electron density as $n(E)=\int_{-\infty}^{E} \tilde{\rho}(E) d E-n_{\text {ions }}$, where $n_{\text {ions }}=3.9 \cdot 10^{15} \mathrm{~cm}^{-2}$ is the density of the positive ions in the graphene lattice compensating the negative charge of the $p$-electrons [note that for an ideal graphene lattice at the neutrality point $n(E)=0$ ]. Combining the calculated $n(E)$ with $\sigma(E)$ given by Eq. (6) we compute the density dependence of the conductivity $\sigma=\sigma(n)$. Details of numerical calculations of DOS, $D(E, t)$, and $\sigma$ are given in Ref. 5 .

\section{RESULTS AND DISCUSSION}

This section contains numerical results for the dc conductivity calculated using the time-dependent real space Kubo-Greenwood formalism within the tight-binding model briefly presented in the previous section. We perform calculations for three different concentrations of $1 \mathrm{D}$ defects corresponding to 10,50 , and 100 line defects in the $1700 \times 1000$-size lattice (see Fig. 1). Parameters $A$ and $B$ entering the scattering potential are weakly electron-density dependent. By fitting the analytical expression for the Thomas-Fermi potential to the Lorentzian-type one, Eq. (2) $\underline{\underline{18}}$ in the range of representative electron densities $1 \cdot 10^{-5} \lesssim\left|n_{e}^{\exp }\right| \lesssim 5 \cdot 10^{-5}$ atom $^{-1}$ used in the present study, we obtain $A=33.57$ and $B=16.96$. All calculations for both symmetric, $V \gtrless 0$, and asymmetric, $V>0$, potentials are performed for the potential strength $\Delta=0.25 u=0.675 \mathrm{eV}$, which 

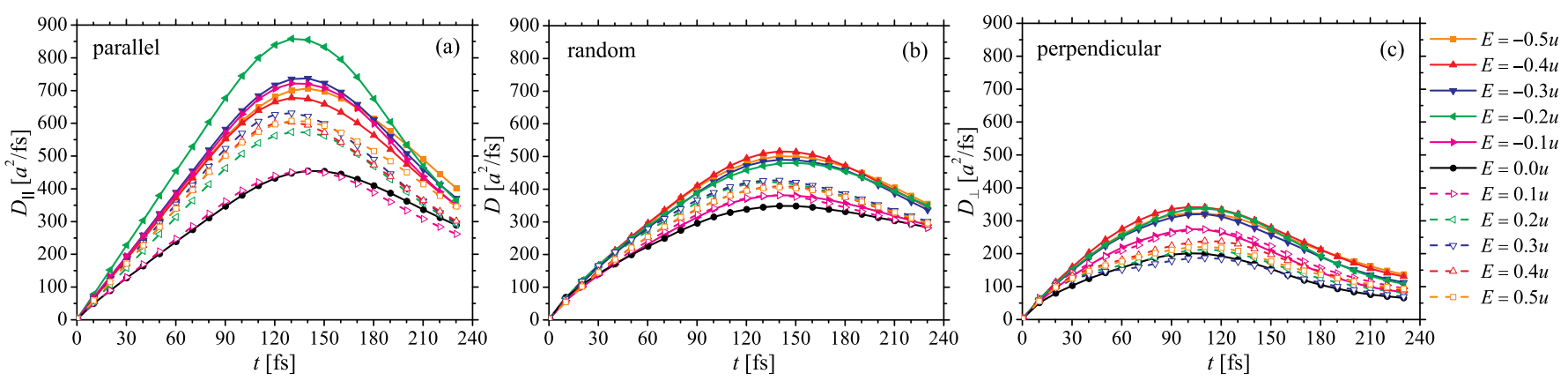

Figure 2: (Color online) Time-dependent diffusion coefficients within the energy interval $E \in[-0.5 u, 0.5 u]$ for 50 line defects, which are (a) parallel $\left(D_{\|}\right)$, (b) randomly distributed $\left(D \equiv D_{\text {rnd }}\right)$, or (c) perpendicular $\left(D_{\perp}\right)$ to the direction of transport (i.e. $x$-direction) (see Fig. 1). $V \gtrless 0, \Delta=0.25 u$.

is close to the values of the contact potential variation at the substrate atomic steps observed in epitaxial graphene by means of the Kelvin probe force microscopy $7,10,11$

Figure 2 shows the time evolution of the diffusion coefficient, Eq. (5), within the energy interval $E \in$ $[-0.5 u, 0.5 u]$ for the symmetric potential, $V \gtrless 0$, for three different cases of the orientational distribution of 50 line defects. In the first and the third cases, Figs. 2 (a) and 2(c), the diffusion coefficients $D_{\|}$and $D_{\perp}$ are calculated respectively along and across 50 parallel-oriented line defects (the distance between lines is different and random). In the second case, Fig. 2(b), the lines are randomly distributed, which results in the isotropic diffusivity, $D_{\text {rnd }} \equiv D_{x x} \equiv D_{y y}$. As expected, the diffusion coefficients along the lines are higher than those across the lines, whereas $D_{\perp}<D_{\text {rnd }}<D_{\|}$. After an initial linear increase corresponding to the ballistic regime, the diffusion coefficients reach their maxima at $t \approx 130,140$, and 110 fs for the "parallel", "random", and "perpendicular" cases, respectively. These values of $D=D_{\max }$ are used to calculate conductivities in Eq. (6). For times $t \gtrsim 130, t \gtrsim 140$, and $t \gtrsim 110 \mathrm{fs}, D(t)$ decreases due to the localization effects. Similar temporal behavior of the diffusivity was established earlier for point ${ }^{5,25,26}$ and line ${ }^{18}$ defects in graphene.

To ascertain possible effects of the anisotropy, we calculated conductivity for five different cases of orientations of the lines. Figure 3 shows conductivities $\sigma_{\|}$and $\sigma_{\perp}$ for lines which are parallel $(\|)$ and perpendicular $(\perp)$ to the $x$-direction, or, in other words, conductivities in the directions parallel and perpendicular to the parallel-oriented line defects. Figure 3 also shows conductivities along the $x$-direction for line defects with different correlation angles, $\sigma_{x x}^{\alpha_{\max }=0^{\circ}}, \sigma_{x x}^{\alpha_{\max }}=45^{\circ}$, and $\sigma_{x x}^{\alpha_{\max }=90^{\circ}}=\sigma_{\text {rnd }}$ (see Fig. 1 for illustration). The minimum value $\alpha_{\max }=0^{\circ}$ corresponds to the case when all lines are parallel in each of 50 realizations, but have different "preferred" direction in the each configuration. The maximum value $\alpha_{\max }=90^{\circ}$ corresponds to random (totally uncorrelated) lines in the each realization. (Note that Fig. 11ilustrates only one of possible realizations of $1 \mathrm{D}$ defects for the chosen values of $\alpha_{\max }$ and the prevail- ing direction.)

For the case of parallel lines the conductivity along them, $\sigma_{\|}$, substantially exceeds the conductivity in the transverse direction, $\sigma_{\perp}$, for both symmetric $(V \gtrless 0)$ and asymmetric $(V>0)$ scattering potentials. For instance, for 50 line defects, the enhancement is up to 3.5 times (see Fig. 3). Such anisotropy is apparently caused by a significantly weaker electron scattering along the line defects as compared to the transverse direction. As mentioned in the introduction, the conductance anisotropy has been revealed in recent experiments,,$\frac{6,14}{1}$ and our calculations are consistent with these results.

Similarly to the behavior of the diffusion coefficient for the case of randomly oriented lines, the corresponding conductivity, $\sigma_{\text {rnd }}=\sigma_{x x}^{\alpha_{\max }=90^{\circ}}$, is also smaller than $\sigma_{\|}$but larger than $\sigma_{\perp}$. Interestingly, the ratio $\left(\sigma_{\|}-\right.$ $\left.\sigma_{\text {rnd }}\right) / \sigma_{\text {rnd }}$ is larger than the ratio $\left(\sigma_{\text {rnd }}-\sigma_{\perp}\right) / \sigma_{\text {rnd }}$ in all the range of electron densities, see Fig. 4. In other words, for the fully random orientation of the line defects, the corresponding conductivity, $\sigma_{\text {rnd }}=\sigma_{x x}^{\alpha_{\max }=90^{\circ}}$, is more close to $\sigma_{\perp}$ than to $\sigma_{\|}$. (Note that $\left|\sigma_{\|}-\sigma_{x x}^{\alpha_{\max }=45^{\circ}}\right| \approx$ $\left|\sigma_{\perp}-\sigma_{x x}^{\alpha_{\max }=45^{\circ}}\right|$.) This feature of the conductivity for the case of the random defect orientation (i.e. $\left|\sigma_{\|}-\sigma_{\text {rnd }}\right| \neq$ $\left.\left|\sigma_{\perp}-\sigma_{\text {rnd }}\right|\right)$ is related to the dominant contribution to the conductivity of the most strong scatterers,-- line defects which are or almost perpendicular to the given direction of transport. When the correlation angle decreases from its maximum value $\left(\alpha_{\max }=90^{\circ}\right)$ through the intermediate one $\left(\alpha_{\max }=45^{\circ}\right)$ to the minimum value $\left(\alpha_{\max }=0^{\circ}\right)$, the conductivity is also gradually decreases for both symmetric $(V \gtrless 0)$ and asymmetric $(V>0)$ potentials (Fig. 3). Note, that this behavior of the conductivity for the line defects can be contrasted with the case of point defects, described by symmetric (attractive and repulsive) scattering potential, when the correlation in the defect position practically does not affect the conductivity.

To ascertain how the conductivity of graphene sheets depends on a concentration of the correlated line defects, we performed numerical calculations for the same computational domain size (1.7 millions of atoms), but with different number $(10,50$, and 100) of line defects randomly distributed and parallel-oriented in each of (20) consid- 

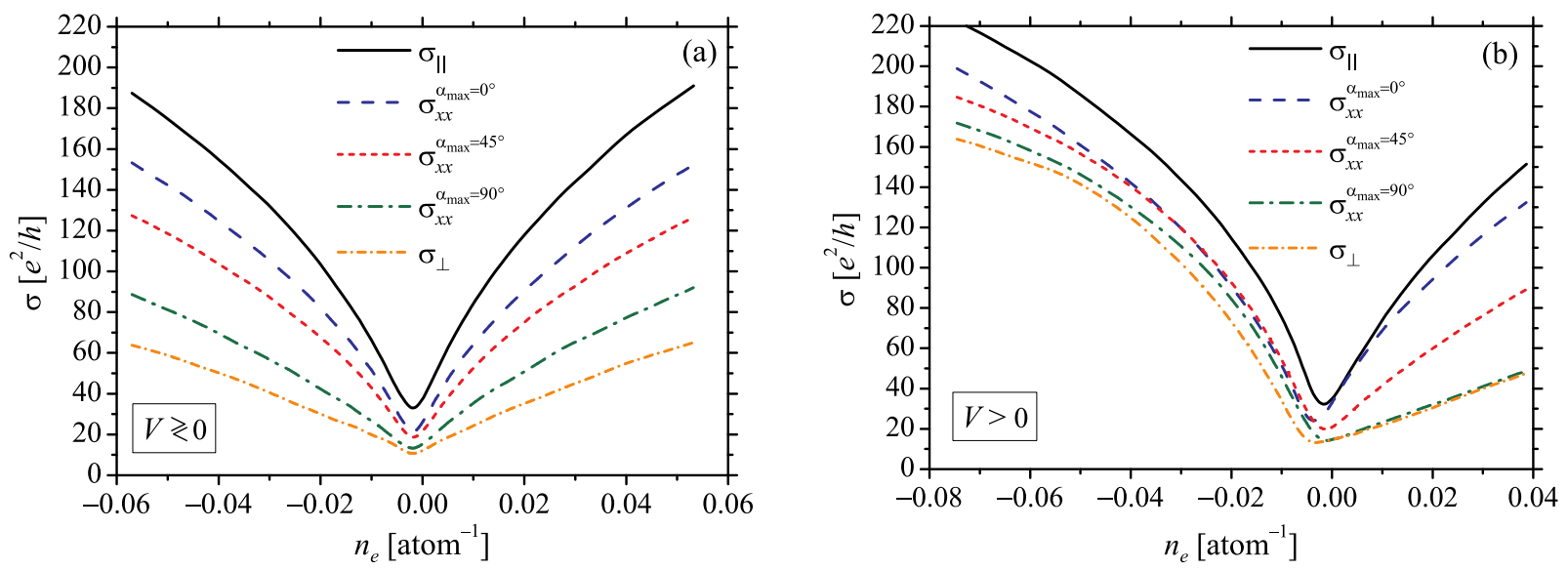

Figure 3: (Color online) Conductivities $\sigma_{x x}^{\alpha_{\max }}$ vs. the relative charge carrier density for (a) symmetric, $V \gtrless 0$, and (b) asymmetric, $V>0$, scattering potentials (with $\Delta=0.25 u$ ) for different configurations of 50 line defects. Each curve averages 50 different defect configurations, corresponding to different distances between the lines and different prevailing directions in the each realization. $\sigma_{\|}$and $\sigma_{\perp}$ are the conductivities in parallel and perpendicular directions to the parallel lines (corresponding to $\left.\alpha_{\max }=0\right)$.
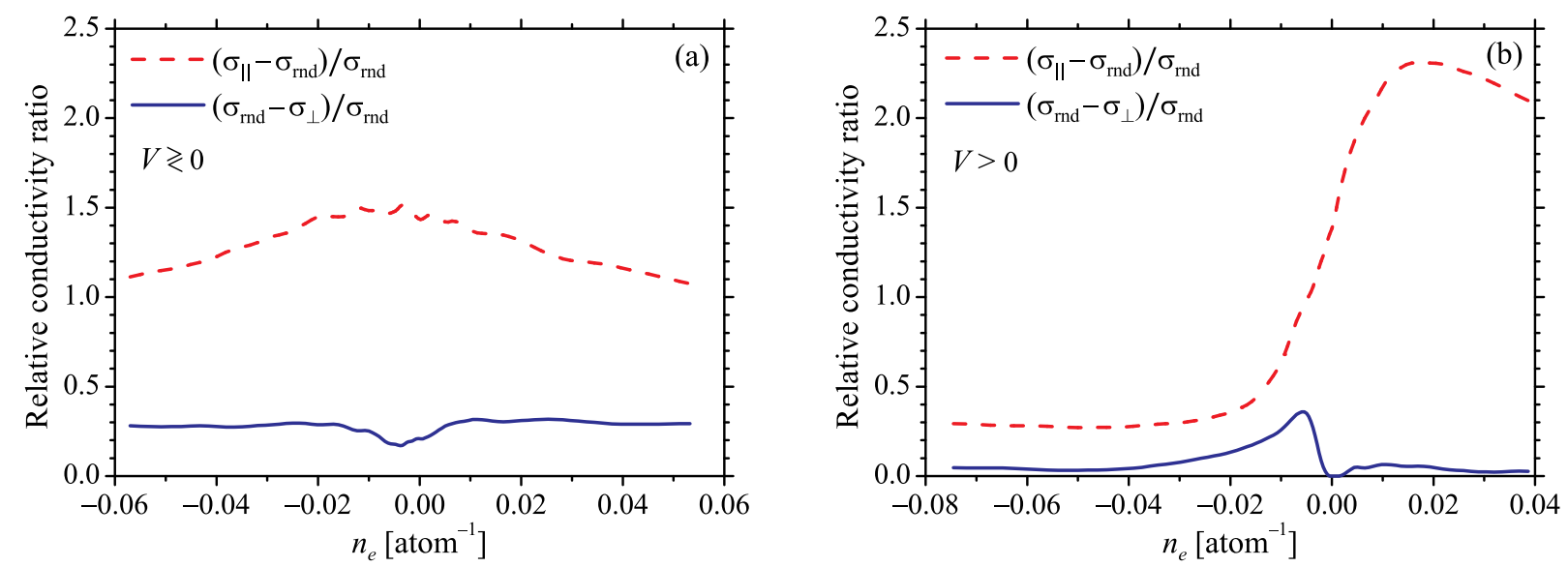

Figure 4: (Color online) The ratios $\left(\sigma_{\|}-\sigma_{\text {rnd }}\right) / \sigma_{\text {rnd }}$ and $\left(\sigma_{\text {rnd }}-\sigma_{\perp}\right) / \sigma_{\text {rnd }}$ representing relative increase (decrease) of the conductivity in the direction parallel (perpendicular) to 50 parallel line defects as compared to the conductivity $\sigma_{\text {rnd }}=\sigma_{x x}^{\alpha_{\max }=90^{\circ}}$ along the $x$-direction for the same number of randomly distributed lines.

ered realizations. As Figs. 5 and 6 show, for a given electron density, the relative increase of the conductivity for the case of fully correlated line defects in comparison to the case of uncorrelated ones is higher for a larger defect density. For example, for the case of the symmetric potential with 10 lines, $\sigma_{x x}^{\alpha_{\max }=0^{\circ}} / \sigma_{x x}^{\alpha_{\max }=90^{\circ}} \approx 1.15$, whereas for the case of 100 lines, $\sigma_{x x}^{\alpha_{\max }=0^{\circ}} / \sigma_{x x}^{\alpha_{\max }=90^{\circ}} \approx$ 2.75 [Fig. [6(a)]; and for the asymmetric potential, ratios $\sigma_{x x}^{\alpha_{\max }=0^{\circ}} / \sigma_{x x}^{\alpha_{\max }=90^{\circ}}$ may reach values up to $\approx 1.35$ and $\approx 5$ for 10 and 100 lines, respectively [Fig. 6(b)]. This is an expected result, because the correlation effect manifests itself stronger for a larger number of objectsto-be-correlated,--line defects in our case.

It is noteworthy that the conductivity exhibits a pronounced sublinear dependence as a function of the electron density. For the case of uncorrelated line defects such atypical behavior has been attributed to the ex- tended nature of one-dimensional charged defects $\underline{\underline{18}}$ Apparently, the sublinear density dependence persists also in the case of correlated extended defects. It should be noted however that when a defect concentration is increased the sublinear density dependence of the conductivity gradually transforms into the linear one, see Fig. 5. Interestingly that with the increase of the defect concentration for the asymmetric potential $(V>0), \sigma(n)$ remains sublinear for $n<0$ and transforms into linear one for $n>0$ (i.e. in the density region where the conductivity is affected most).

In realistic samples one can expect that the conductivity is also affected by the presence of point-like scatterers such as adatoms and charged impurities, $\underline{5,18,24}-26,29,30,34,35$ In the following discussion we focus on the effect of asymmetry of the conductance for the case of asymmetric potentials $(V>0)$. Figure 

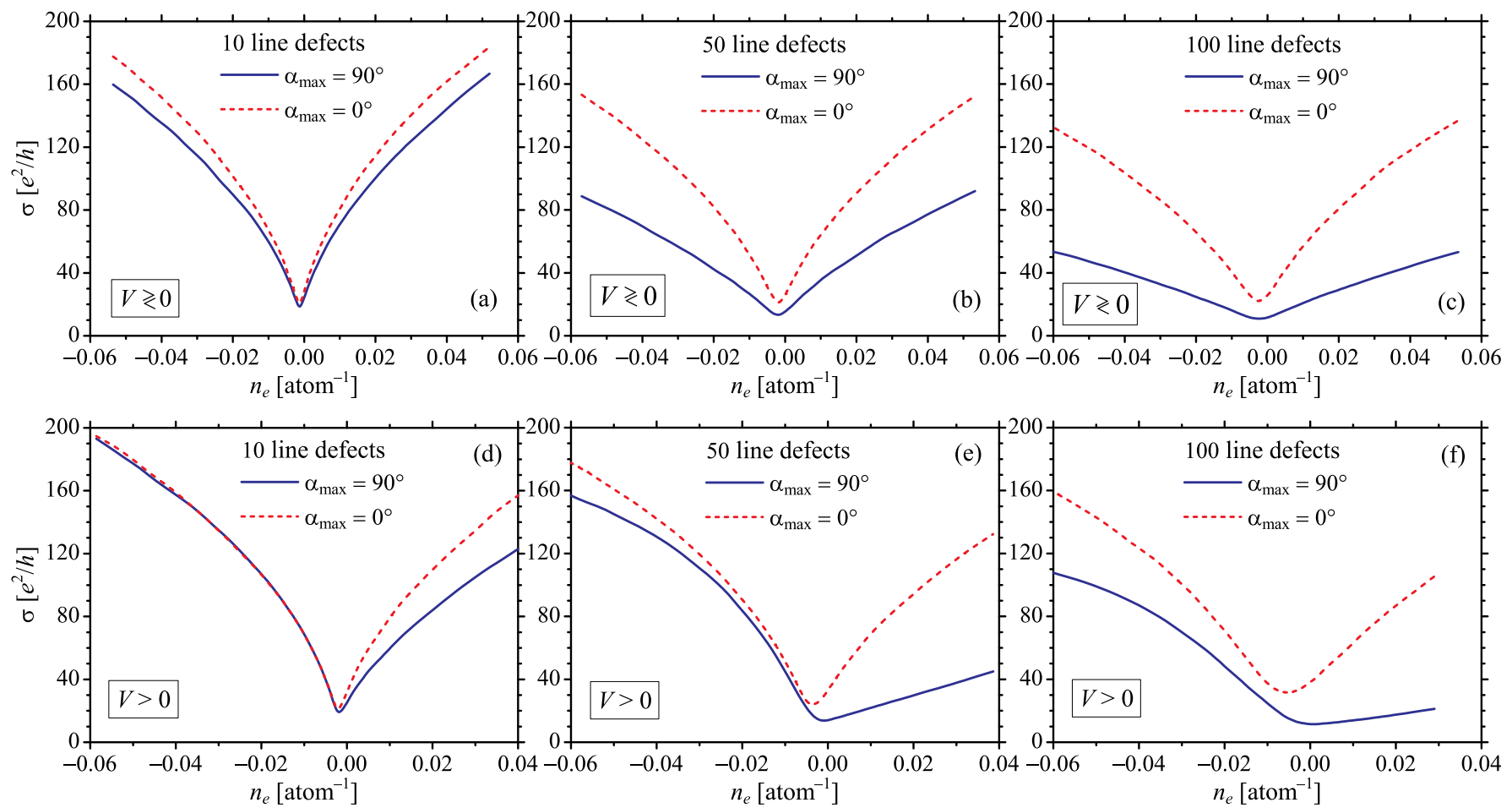

Figure 5: (Color online) Conductivities $\sigma_{x x}^{\alpha_{\max }}$ vs. the relative charge carrier density for (a)-(c) symmetric, $V \gtrless 0$, and (d)-(f) asymmetric, $V>0$, scattering potentials (with $\Delta=0.25 u$ ) for different number $(10,50,100)$ of lines for the cases of $\alpha_{\max }=90^{\circ}$ and $\alpha_{\max }=0^{\circ}$.
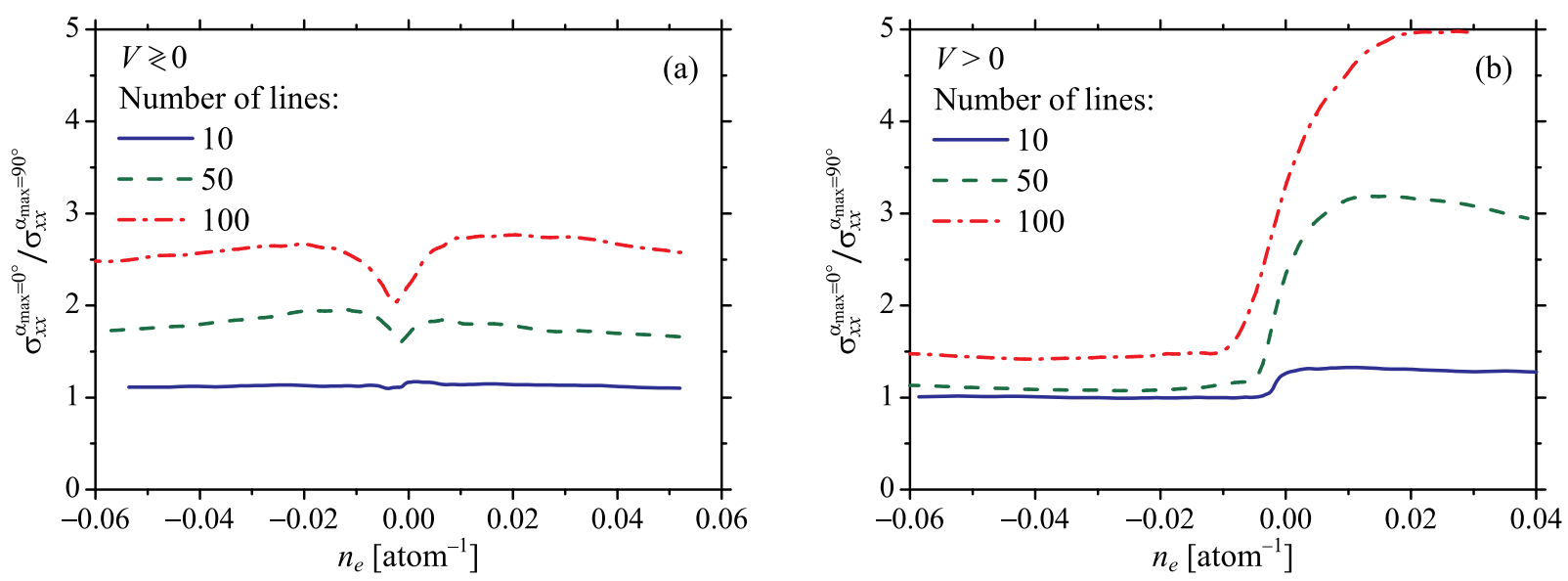

Figure 6: (Color online) Enhancement of the conductivity (in terms of the ratio $\sigma_{x x}^{\alpha_{\max }=0^{\circ}} / \sigma_{x x}^{\alpha_{\max }=90^{\circ}}$ ) due to the correlation of line defects for different number $(10,50$, and 100) of lines for (a) symmetric, $V \gtrless 0$, and (b) asymmetric, $V>0$, scattering potentials.

7 shows the conductivity of graphene sheet for the case when both extended line defects and point-like defects are present. The point-like defects are modeled by the Gaussian potential $V_{\text {imp }}=\sum_{0}^{N_{i}} U_{i} e^{-\mathbf{r}^{2} / 2 \xi^{2}}$, where $U_{i}$ is chosen randomly in the range $[0, \Delta]$, and $\xi$ is the effective potential radius $\stackrel{5,24,30}{ }$ To model short-range scatteres such as neutral adatoms we choose $\xi=0.5 a$ [Figs. 7(a) and 7(c)], and to model long-range charged impurities we choose $\xi=4 a$ [Figs. 7(b) and 7(d)].
For further discussion it is important to stress that conductivity of graphene with point defects with asymmetric potential $(V>0)$ is not symmetric with respect to the Dirac point. (Note that asymmetric behavior of the conductivity for point-like defects was discussed in e.g. Refs. 34, 35.) However, this asymmetry is much more pronounced for the case of short-ranged defects as compared to long-ranged ones [cf. short dashed blue curves in Figs. [7(c) and $7(\mathrm{~d})]$. Let us now consider the case of line 

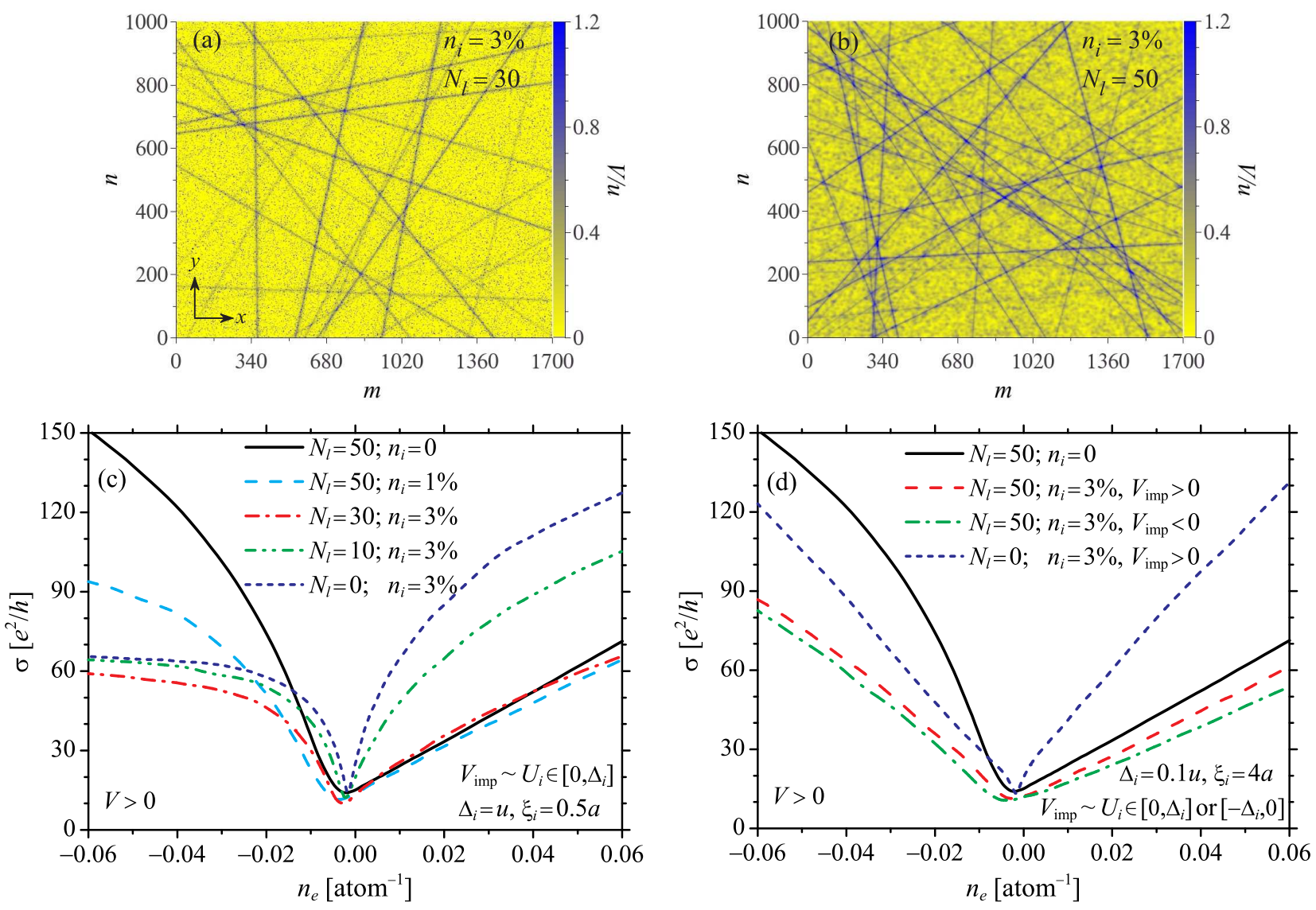

Figure 7: (Color online) (a)-(b) Potential distributions in a graphene sheet of the size $1700 \times 1000$ sites with (a) $N_{l}=30$ line defects and $N_{i}=51000$ short-range point scatterers; and (b) $N_{l}=50$ line defects and $N_{i}=51000$ long-range point scatterers. (c)-(d) The density-dependent conductivity for the graphene sheets with (c) line defects and short-range point defects, and (d) line defects and long-range point defects. The line defects are described by the long-range Lorentzian $V$ (2), while the point scatterers are modeled by the Gaussian $V_{\mathrm{imp}}=\sum_{0}^{N_{i}} U_{i} e^{-\mathbf{r}^{2} / 2 \xi_{i}^{2}}$ with effective potential radius (a), (c) $\xi_{i}=0.5 a$ and (b), (d) $\xi_{i}=4 a ; U_{i}$ chosen randomly in the range $\left[0, \Delta_{i}\right]$.

defects + short-range point defects [Figs. 7(a) and 7(c)]. Each type of scatterers alone exhibits strong asymmetric behavior of the opposite symmetry [cf. black solid and blue short-dashed curves in Fig. [7(c)]. Therefore, depending on the relative concentration of the line- and point defects, the conductivity can be enhanced whether for positive or negative charge densities, see Fig. 7(c). In contrast, for the case of line defects + long-range point defects a character of the asymmetry of the conductivity is different. In this case, because of the weak asymmetry of the density dependence of $\sigma$ for the long-range point defects, the asymmetry of the conductivity of the graphene is dominated by the asymmetry due to the line defects regardless of the sign of the scattering potential of point scatterers, see Fig. 7(d).

\section{CONCLUSIONS}

A numerical study of the conductivity of graphene with correlated extended line defects is performed using the ef- ficient time-dependent real-space Kubo-Greenwood formalism. The scattering by the line defects was modeled by an effective long-ranged potential of the Lorentzian shape. The correlation in the spatial distribution of the line defects was described by the correlation angle $\alpha_{\max }$ (i.e. the maximum possible angle between any two lines). For the case of parallel lines $\left(\alpha_{\max }=0\right)$, we find that the conductivity along the lines, $\sigma_{\|}$, substantially exceeds the conductivity in the transverse direction, $\sigma_{\perp}$, which agrees well with the experimental measurements for epitaxial graphene. We found that for a given electron density the relative increase of the conductivity for the case of the totally correlated line defects in comparison to the case of uncorrelated ones is higher for a larger defect density. We also discuss a combined effect of extended line defects and point short- and long-range defects focusing on the character of the asymmetry of the conductivity with respect to the Dirac point. 


\section{Acknowledgments}

The authors greatly appreciate discussions with Chariya Virojanadara, Rositsa Yakimova and Volodymyr Khranovskyy concerning the structure and type of defects in epitaxial graphene and acknowledge Stephan Roche for discussion about the time-dependent Kubo-
Greenwood approach. T.M.R. benefited immensely from discussions with Sergei Sharapov and also expresses gratitude to Valentyn Tatarenko for frequent discussions on various short- and long-range scattering and interaction potentials. The authors acknowledge support from the Swedish Institute.
${ }^{1}$ K. S. Novoselov, V. I. Fal'ko, L. Colombo, P. R. Gellert, M. G. Schwab, and K. Kim, Nature 490, 11 (2012).

${ }^{2}$ F. Banhart, J. Kotakovski, and A. V. Krasheninnikov, ACS Nano 5, 26 (2011).

3 Jun Yan and M. S. Fuhrer, Phys. Rev. Lett. 107, 206601 (2011).

4 Qiuzi Li, E. H. Hwang, E. Rossi, and S. Das Sarma, Phys. Rev. Lett. 107, 156601 (2011).

5 T. M. Radchenko, A. A. Shylau, and I. V. Zozoulenko, Phys. Rev. B 86, 035418 (2012).

${ }^{6}$ H. Kuramochi, S. Odaka, K. Morita, S. Tanaka, H. Miyazaki, M. V. Lee, S.-L. Li, H. Hiura, and K. Tsukagoshi, AIP Advances 2, 012115 (2012)

7 Ch. Held, T. Seyller, and R. Bennewitz, Beilstein J. Nanotechnol. 3, 179 (2012).

8 S. Günther, S. Dänhardt, B. Wang, M.-L. Bocquet, S. Schmitt, and J. Wintterlin, Nano Lett. 11, 1895 (2011).

9 W. Melitz, J. Shen, A. C. Kummel, and S. Lee, Surf. Sci. Rep. 66, 1 (2011).

10 S.-H. Ji, J. B. Hannon, R. M. Tromp, V. Perebeinos, J. Tersoff, and F. M. Ross, Nature Mater. 11, 114 (2012).

11 W. Wang, K. Munakata, M. Rozler, and M. R. Beasley, Phys. Rev. Lett. 110, 236802 (2013).

12 C. Dimitrakopoulos, A. Grill, T. McArdle, Z. Liu, R. Wisnieff, and D. A. Antoniadis, Appl. Phys. Lett. 98, 222105 (2011).

13 Y. M. Lin, D. B. Farmer, K. A. Jenkins, Y. Wu, J. L. Tedesco, R. L. Myers-Ward, C. R. Eddy, D. K. Gaskill, C. Dimitrakopoulos, and P. Avouris, IEEE Electron Device Lett., 32, 1343 (2011).

14 M. K. Yakes, D. Gunlycke, J. L. Tedesco, P. M. Campbell, R. L. Myers-Ward, Ch. R. Eddy, Jr., D. K. Gaskill, P. E. Sheehan, and A. R. Laracuente, Nano Lett. 10, 1559 (2010).

15 T. Low, V. Perebeinos, J. Tersoff, and Ph. Avouris, Phys. Rev. Lett. 108, 096601 (2012).

16 G.-X. Ni, Y. Zheng, S. Bae, H. R. Kim, A. Pachoud, Y. S. Kim, Ch.-L. Tan, D. Im, J.-H. Ahn, B. H. Hong, and B. Özyilmaz, ACS Nano 6, 1158 (2012).

17 A. Ferreira, X. Xu, C.-L. Tan, S.-K. Bae, N. M. R. Peres, B.-H. Hong, B. Ozyilmaz, and A. H. Castro Neto, EPL,
94, 28003 (2011).

18 T. M. Radchenko, A. A. Shylau, I. V. Zozoulenko, and A. Ferreira, Phys. Rev. B 87, 195448 (2013).

19 D. V. Tuan, J. Kotakoski, T. Louvet, F. Ortmann, J. C. Meyer, and S. Roche, Nano Lett. 13, 1730 (2013).

20 S. Ihnatsenka and I. V. Zozoulenko, Phys. Rev. B 88, 085436 (2013).

21 O. V. Yazyev and S. G. Louie, Nat. Mater 9, 806 (2010).

22 S. Roche, N. Leconte, F. Ortmann, A. Lherbier, D. Soriano, and J.-Ch. Charlier, Solid State Comm. 153, 1404 (2012).

23 T. Markussen, R. Rurali, M. Brandbyge, and A.-P. Jauho, Phys. Rev. B 74, 245313 (2006); T. Markussen, Master thesis, Technical University of Denmark, 2006.

${ }^{24}$ S. Yuan, H. De Raedt, and M. I. Katsnelson, Phys. Rev. B 82, 115448 (2010).

${ }^{25}$ N. Leconte, A. Lherbier, F. Varchon, P. Ordejon, S. Roche, and J.-C. Charlier, Phys. Rev. B 84, 235420 (2011).

26 A. Lherbier, Simon M.-M. Dubois, X. Declerck, Y.-M. Niquet, S. Roche, and J.-Ch. Charlier, Phys. Rev. B 86, 075402 (2012)

27 H. Ishii, N. Kobayashi, and K. Hirose, Phys. Rev. B 82, 085435 (2010).

28 A. H. Castro Neto, F. Guinea, N. M. R. Peres, K. S. Novoselov, and A. K. Geim, Rev. Mod. Phys. 81, 109 (2009).

29 N. M. R. Peres, Rev. Mod. Phys. 82, 2673 (2010).

30 S. Das Sarma, S. Adam, E. H. Hwang, and E. Rossi, Rev. Mod. Phys. 83, 407 (2011).

31 A. Mattausch and O. Pankratov, Phys. Rev. Lett. 99, 076802 (2007).

${ }^{32}$ F. Varchon, R. Feng, J. Hass, X. Li, B. N. Nguyen, C. Naud, P. Mallet, J.-Y. Veuillen, C. Berger, E. H. Conrad, and L. Magaud, Phys. Rev. Lett. 99, 126805 (2007).

33 O. Madelung, Introduction to Solid-State Theory (Springer, Berlin, 1996).

34 J. P. Robinson, H. Schomerus, L. Oroszlany, and V. I. Fal'ko, Phys. Rev. Lett. 101, 196803 (2008).

35 T. O. Wehling, S. Yuan, A. I. Lichtenstein, A. K. Geim, and M. I. Katsnelson, Phys. Rev. Lett. 105, 056802 (2010). 